\title{
Randomized controlled trial of remote ischaemic conditioning in ST-elevation myocardial infarction as adjuvant to primary angioplasty (RIC-STEMI)
}

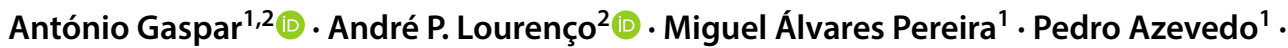 \\ Roberto Roncon-Albuquerque Jr. ${ }^{2}\left[\right.$ (D) Jorge Marques ${ }^{1} \cdot$ Adelino F. Leite-Moreira $^{2}(\mathbb{C}$
}

Received: 25 February 2018 / Revised: 1 March 2018 / Accepted: 2 March 2018 / Published online: 7 March 2018

(c) Springer-Verlag GmbH Germany, part of Springer Nature 2018

\begin{abstract}
To test whether remote ischaemic conditioning (RIC) as adjuvant to standard of care (SOC) would prevent progression towards heart failure (HF) after ST-elevation myocardial infarction (STEMI). Single-centre parallel 1:1 randomized trial (computerized block-randomization, concealed allocation) to assess superiority of RIC (3 cycles of intermittent 5 min lower limb ischaemia) over SOC in consecutive STEMI patients (NCT02313961, clinical trials.gov). From 258 patients randomized to RIC or SOC, 9 and 4\% were excluded because of unconfirmed diagnosis and previously unrecognized exclusion criteria, respectively. Combined primary outcome of cardiac mortality and hospitalization for HF was reduced in RIC compared with SOC ( $n=231$ and 217, respectively; HR $=0.35,95 \%$ CI 0.15-0.78) as well as each outcome in isolation. No difference was found in serum troponin I levels between groups. Median and maximum follow-up time were 2.1 and 3.7 years, respectively. In-hospital HF $(\mathrm{RR}=0.68,95 \% \mathrm{CI} 0.47-0.98)$, need for diuretics ( $\mathrm{RR}=0.68,95 \% \mathrm{CI} 0.48-0.97)$ and inotropes and/or intra-aortic balloon pump ( $R R=0.17,95 \%$ CI 0.04-0.76) were decreased in RIC. On planned 12 months follow-up echocardiography ( $n=193$ and 173 in RIC and SOC, respectively) ejection fraction (EF) recovery was enhanced in patients presenting with impaired left ventricular (LV) function ( $10 \%$ absolute difference in median EF compared with SOC; $P<0.001)$. In addition to previously reported improved myocardial salvage index and reduced infarct size RIC was shown beneficial in a combined hard clinical endpoint of cardiac mortality and hospitalization for HF. Improved EF recovery was also documented in patients with impaired LV function.
\end{abstract}

Keywords ST-elevation myocardial infarction $\cdot$ Remote ischaemic conditioning $\cdot$ Heart failure $\cdot$ Randomized controlled trial $\cdot$ Left ventricular function

\begin{tabular}{|c|c|c|c|c|}
\hline \multicolumn{3}{|c|}{ Abbreviations } & CABG & Coronary artery bypass grafting \\
\hline \multirow{2}{*}{\multicolumn{2}{|c|}{ ACEi/ARB }} & Angiotensin-converting enzyme inhibitor or & $\mathrm{CI}$ & Confidence intervals \\
\hline & & angiotensin II receptor blocker & CIN & Contract-induced nephropathy \\
\hline \multicolumn{2}{|c|}{ AUC } & Area under the curve & CRT & Cardiac resynchronization therapy \\
\hline \multicolumn{2}{|c|}{ BMI } & Body mass index & $\mathrm{EF}$ & Ejection fraction \\
\hline \multicolumn{2}{|c|}{$\mathrm{BP}$} & Blood pressure & $\mathrm{HB}$ & Hospital de Braga \\
\hline & $\mathrm{HF}$ & Heart failure \\
\hline \multirow{3}{*}{\multicolumn{3}{|c|}{$\begin{array}{l}\text { A comment to this article is available at https://doi.org/10.1007/ } \\
\text { s00395-018-0673-2. }\end{array}$}} & HR & Hazard ratio \\
\hline & & & IABP & Intra-aortic balloon pump \\
\hline & & & ICD & Implantable cardioverter-defibrillator \\
\hline & \multirow{2}{*}{\multicolumn{2}{|c|}{$\begin{array}{l}\text { Adelino F. Leite-Moreira } \\
\text { amoreira@ med.up.pt }\end{array}$}} & IHD & Ischaemic heart disease \\
\hline & & & IRI & Ischaemia-reperfusion injury \\
\hline \multirow[t]{2}{*}{1} & \multirow{2}{*}{\multicolumn{2}{|c|}{$\begin{array}{l}\text { Department of Cardiology, Hospital de Braga, Braga, } \\
\text { Portugal }\end{array}$}} & ITT & Intention-to-treat \\
\hline & & & LV & Left ventricle \\
\hline \multirow[t]{2}{*}{2} & \multirow{2}{*}{\multicolumn{2}{|c|}{$\begin{array}{l}\text { Department of Surgery and Physiology, Cardiovascular R\&D } \\
\text { Unit, Faculty of Medicine, University of Porto, Alameda } \\
\text { Prof. Hernâni Monteiro, 4200-319 Porto, Portugal }\end{array}$}} & MACCE & $\begin{array}{l}\text { Major adverse cardiovascular and cerebro- } \\
\text { vascular events }\end{array}$ \\
\hline & & & MI & Myocardial infarction \\
\hline
\end{tabular}




$\begin{array}{ll}\text { PCI } & \text { Percutaneous coronary intervention } \\ \text { RCT } & \text { Randomized controlled trial } \\ \text { RIC } & \text { Remote ischaemic conditioning } \\ \text { RR } & \text { Risk ratio } \\ \text { SOC } & \text { Standard of care } \\ \text { STEMI } & \text { ST-elevation myocardial infarction } \\ \text { TIMI } & \text { Thrombolysis in myocardial infarction study } \\ & \text { group grading of coronary flow }\end{array}$

\section{Introduction}

Ischaemic heart disease (IHD) and myocardial infarction (MI) remain the leading cause of mortality in Europe [22]. ST-elevation myocardial infarction (STEMI) incidence declined due to aggressive control of risk factors while mortality was reduced by combined effects of timely primary percutaneous coronary intervention (PCI) and optimized medical therapy $[4,10]$. An increasing number of survivors, however, are at risk of left ventricular (LV) dysfunction [17]. PCI worsens ischaemia-reperfusion injury (IRI), which later accounts for larger infarct size and ensuing heart failure (HF) [7]. Indeed, 6-month mortality figures after STEMI remain high, warranting a shift in focus towards HF prevention [6]. The rationale underlying protection by ischaemic conditioning has been extensively reviewed [11, 14], but translation to the clinics has been unwieldy $[10,12]$. From various alternatives remote ischaemic conditioning (RIC) seems the most promising [10]. Myocardial salvage index was improved 30 days after PCI in STEMI patients assigned to concomitant RIC [2] as was long-term prognosis in post hoc analysis [21]. A recent meta-analysis concluded that RIC is a promising adjunctive treatment to PCI for prevention of IRI in STEMI [18]. Most importantly, larger studies addressing hard clinical endpoints as primary outcome measures are warranted [10].

Our aim was to test the hypothesis that RIC as adjuvant therapy to standard of care PCI (SOC) could reduce a combined primary outcome measure of cardiac mortality and hospitalization for HF on follow-up after STEMI.

\section{Methods}

\section{Trial design}

We conducted a single-centre, open-label, parallel 1:1 randomized controlled trial (RCT) to assess superiority of RIC as an adjunctive therapy to SOC in consecutive all-comer patients presenting with STEMI between March 2013 and December 2015 (RIC-STEMI). The study was approved by our Institution's Ethical Committee and was conducted in accordance with the Declaration of Helsinki
1964 as revised in 2013 and the International Conference of Harmonisation Guidelines for Good Clinical Practice. All patients gave written informed consent. The protocol was published [8] and registered prospectively in clinicaltrials.gov (NCT02313961). No major changes to design were implemented. Patient enrolment went as planned.

Eligible patients were $\geq 18$ years old, presenting to the emergency department of Hospital de Braga (HB) with putative STEMI defined as chest (or epigastric) pain $>30$ min within $12 \mathrm{~h}$ of onset and either (1) new ST-elevation at the J point in 2 contiguous leads (cut-off points of $\geq 0.2$ and $\geq 0.15 \mathrm{mV}$ in men and women, respectively) in leads V2-V3 or $\geq 0.1 \mathrm{mV}$ in other leads; (2) or new left bundle branch block. Exclusion criteria were: cardiogenic shock as defined by systolic blood pressure (BP) $<90 \mathrm{mmHg}$ and evidence of tissue hypoperfusion, post-cardiac arrest status, need for mechanical ventilation, known peripheral artery disease or evidence of lower limb ischaemia, and recent MI (within 30 days). Eligible patients were first enrolled based on presumed diagnosis and witnessed oral consent. Written consent was obtained under stable medical conditions as soon as possible.

\section{Intervention}

Participants were randomized 1:1 to adjunctive therapy with RIC or SOC in blocks of 4 according to a computergenerated list. Randomization list was held back at the department and accessed by an assigned nurse that was previously unaware of allocation. Intervention consisted of placement of a BP cuff in the left lower limb upon arrival to the cardiac catheterization laboratory. Attending physicians and patients were unaware of allocation status but were not blinded. RIC was begun 10 min before the estimated time of first balloon inflation. Ischaemia was induced by 3 cycles of inflation and deflation of the cuff to 200 and $0 \mathrm{mmHg}$, respectively, for $5 \mathrm{~min}$ each (inflation and deflation). Patients allocated to SOC did not have their cuffs inflated. SOC consisted of $250 \mathrm{mg}$ intravenous aspirin, an oral $\mathrm{P} 2 \mathrm{Y}_{12}$ inhibitor and 5000 IU intravenous unfractionated heparin according to institutional guidelines. The choice of balloons, stents and procedure as well as the use of glycoprotein IIb/IIIa inhibitors were left to the discretion of attending physicians. Radial arteries were the preferred access for cardiac catheterization. According to the institution's practice intracoronary vasodilators are not used except for nitrates in cases of slow flow. Patients were excluded from further analysis if STEMI was not confirmed on coronarography. Venous samples were collected serially at 12-h intervals from admission to $48 \mathrm{~h}$. Patient data were anonymized and group allocation was veiled as of the intervention. 


\section{Outcomes}

The primary outcome measure was combined mortality from cardiac cause or hospitalization for HF on followup. Minimum predefined follow-up time was 12 months. Secondary outcome measures were 48 -h area under curve (AUC) of serum troponin I levels (trapezoidal rule), ejection fraction (EF) on follow-up (estimated by Simpson's biplane method), contrast-induced nephropathy (CIN), and all-cause mortality, cardiac mortality, hospitalization for $\mathrm{HF}$ and major adverse cardiovascular and cerebrovascular events on follow-up (MACCE). CIN was defined as impaired renal function denoted by either $>25 \%$ increase or an absolute increase $>0.5 \mathrm{mg} / \mathrm{dL}$ in serum creatinine after cardiac catheterization Hospitalization for $\mathrm{HF}$ included readmissions due to acute or chronically decompensated HF (NYHA class > II) or planned implantation of cardioverter-defibrillator (ICD) or cardiac resynchronization therapy device (CRT). Admission was of the sole responsibility of attending physicians from HB or neighbouring institutions. Diagnosis of decompensated HF was confirmed from electronic health records in every patient by NYHA class worsening and raised natriuretic peptide levels. MACCE was defined as the composite of death, MI, stroke, or target vessel revascularization. Stroke was presumed from persistent neurologic deficits. Data were obtained through patient visits, medical records and structured phone interviews. Follow-up echocardiography was scheduled for 12 months post-STEMI. Cause of death was assigned and reviewed blindly by 3 consulting cardiologists and 2 independent researchers, respectively. Cardiac death was defined as natural death due to cardiac causes and required evidence of cardiogenic shock, acutely decompensated heart failure or sudden cardiac arrest. All cases in which there was a reasonable doubt regarding cause of death were not considered as cardiac. Patients found dead at home were not considered to have died of cardiac cause. Database was audited and double-checked by two independent researchers. Allocation of patients to group was only revealed when the database was deemed to be complete.

\section{Sample size}

Sample size was estimated as 246 in each group admitting: 36 months accrual period, minimum follow-up of 12 months, primary outcome incidence of $14 \%$ (data were obtained from HB registries) and treatment effect of $40 \%$ (arbitrarily set as relevant) with two-tailed type I error set at 0.05 and power at 0.80 . Estimates took under consideration $8 \%$ cases of unconfirmed STEMI (data were obtained from HB registries), 5\% drop-outs and 5\% losses to follow-up.

\section{Statistical analysis}

Statistical analysis was carried out by an independent researcher blinded to the specific groups under evaluation (randomly coded as binary variable) using IBM $^{\circledR}$ SPSS $^{\circledR}$ software version 23 and $\mathrm{R}$ version 3.4.1. Normality of distribution was checked (Shapiro-Wilk's test). Categorical and continuous variables are: count (percentage) and median (interquartile range), respectively. Continuous variables were not normally distributed. Chi-square test was used to compare categorical variables (Yate's continuity correction for expected counts per category $<5$ ), Kendall's tau-c and Mann-Whitney's test were used to compare ordinal and continuous variables, respectively. Because normal distribution of residuals and homogeneity of variances were violated, follow-up EF data were compared after aligned rank transformation for nonparametric repeated-measures ANOVA [24] with R package https://github.com/mjskay/ARTool. A similar approach was undertaken for TIMI flow grade before and after angioplasty. Risk ratios (RR) were computed for binomial outcomes along with their $95 \%$ confidence intervals (CI). Cox's proportional hazards model with Breslow's method for ties was employed to compute cumulative hazard of time-to-event outcome measures. Results are reported as hazard ratio (HR) and 95\% CI. Patient subgroup analyses were performed for the main outcome as pre-specified.

\section{Results}

Figure 1 shows the trial profile. We assessed 593 patients for eligibility at arrival to the catheterization laboratory and 516 were randomized. All of the 258 patients randomly allocated to RIC or SOC received the predefined intervention. Later, 45 patients (9\%) were excluded because STEMI diagnosis was not confirmed and 21 (4\%) due to previously unrecognized exclusion criteria. Alternative diagnoses were Takotsubo cardiomyopathy (10 and 8 patients in SOC and RIC, respectively), myocarditis or pericarditis (5 and 3 patients in SOC and RIC, respectively), non-ST elevation MI (6 and 3 patients in SOC and RIC, respectively) and other (5 patients in each group). Previously unrecognized exclusion criteria were cardiogenic shock (4 SOC and 2 RIC patients), postcardiac arrest status (3 SOC and 2 RIC patients) and symptoms with over $12 \mathrm{~h}$ of duration (6 SOC and 4 RIC patients). No difference between groups was found in the proportion of excluded patients ( 10 vs $15 \%, P=0.12$ ). Finally, 2 patients were lost to follow-up in SOC (1\%) due to emigration. Analysed samples consisted of 231 and 217 patients in RIC and SOC, respectively. Patients undergoing RIC experienced no adverse effect besides mild-moderate local discomfort.

RIC and SOC were well-balanced for baseline characteristics (Table 1), except for higher concentrations of 


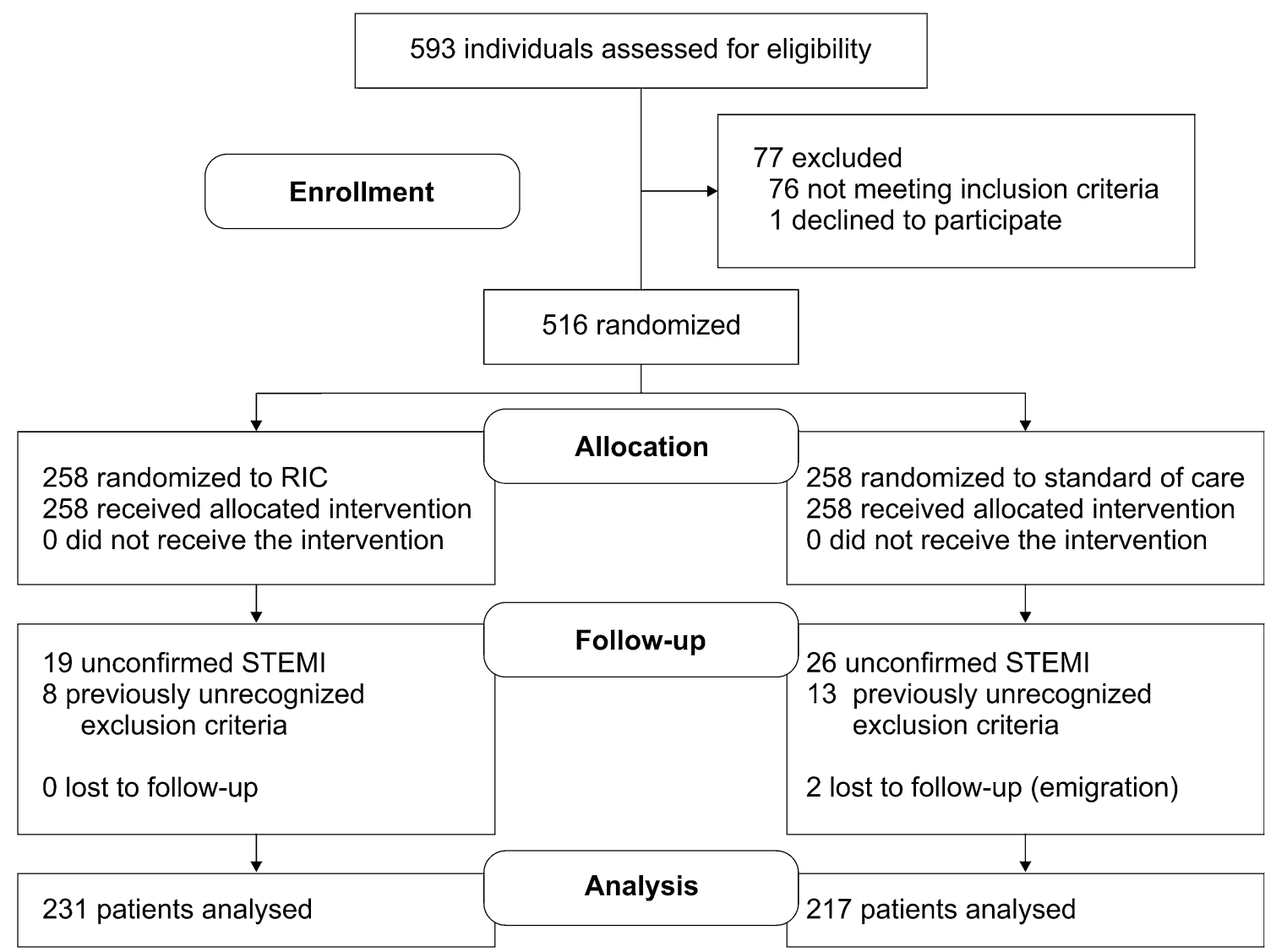

Fig. 1 Study flowchart

haemoglobin in RIC. There were also no procedural differences at the catheterization laboratory (Table 2). No interaction was found between group and change in TIMI flow grade after angioplasty $(P=0.70)$.

During hospital stay, no divergence was found for $48-\mathrm{h}$ area under curve of serum troponin I levels or its peak levels between RIC and SOC (Fig. 2a). No differences were found also for occurrence of malignant arrhythmias, complete atrioventricular block, post-MI angina or recurrent MI (data not shown). All-cause mortality did not differ between RIC and SOC (Fig. 2b). Only 2 SOC patients died from cardiac cause. RIC had no effects on CIN whilst it reduced the risk of HF development as well as the need for diuretics or intra-aortic balloon pump (IABP) and/or inotropes during hospital stay (Fig. 2b). Of note, cardiogenic shock (Killip IV) was observed in 10 SOC and only 2 RIC patients, and levosimendan and IABP were employed in only 3 and 1 SOC cases, respectively. No differences were found for in-hospital medication: aspirin (98\%), second antiplatelet agent (94\%), statins (98\%), $\beta$-blockers (90\%), angiotensin-converting enzyme inhibitors/angiotensin 2 receptor blockers $(92 \%)$ or aldosterone antagonists $(23 \%$, for the pooled sample).
EF was retrieved from follow-up echocardiography in 173 and 193 SOC and RIC patients. By this time 14 and 12 patients from SOC and RIC had died, respectively. Data from 29 (14\%) and 26 (12\%) additional patients from SOC and RIC could not be obtained because patients failed to attend their appointed exam, respectively. No difference was found for missing data count between groups $(P=0.55)$. RIC showed a non-significant trend for improved recovery of EF (Fig. 2, left panel c). Of note, data suggested a distinct pattern of behaviour between patients presenting with higher and lower values of EF which was confirmed by adding 2-quantiles as covariates to the model. Analysis of subgroups revealed a strong interaction consisting of improved EF on follow-up in RIC compared with SOC in patients presenting with lower EF (Fig. 2, right panel c). Median follow-up EF in the lower quantile of EF was 55\% in RIC and $10 \%$ lower in SOC 12 months after STEMI. Interestingly, no differences between groups were found on end-diastolic volume evolution from original admission to follow-up regardless of $\mathrm{EF}$ at presentation. End-diastolic volumes raised from $113(102-130)$ and $118(102-130)$ at admission to 130 (113-147) and $130(108-147) \mathrm{mL}$ at follow-up in SOC and RIC, respectively. 
Table 1 Baseline characteristics

\begin{tabular}{|c|c|c|c|}
\hline & $\operatorname{SOC}(n=217)$ & $\operatorname{RIC}(n=231)$ & $P$ value \\
\hline Age, years & $61(53-72)$ & $59(49-70)$ & 0.10 \\
\hline Female & $50(23)$ & $39(17)$ & 0.10 \\
\hline BMI, $\mathrm{kg} / \mathrm{m}^{2}$ & $26.2(24.4-28.4)$ & $26.6(24-29.4)$ & 0.185 \\
\hline Systemic hypertension & 107 (49) & $113(49)$ & 0.93 \\
\hline Diabetes mellitus & $66(30)$ & $59(26)$ & 0.25 \\
\hline Hypercholesterolemia & $113(52)$ & $111(48)$ & 0.40 \\
\hline Smoker & $123(57)$ & $141(61)$ & 0.35 \\
\hline Previous IHD & $26(12)$ & $26(11)$ & 0.81 \\
\hline Previous PCI and/or CABG & $15(7)$ & $17(7)$ & 0.85 \\
\hline \multicolumn{4}{|l|}{ Ongoing medication } \\
\hline Aspirin & $29(13)$ & $32(14)$ & 0.88 \\
\hline Second antiplatelet agent & $7(3)$ & $10(4)$ & 0.54 \\
\hline Statin & $60(28)$ & $71(31)$ & 0.47 \\
\hline$\beta$-blocker & $35(16)$ & $33(14)$ & 0.59 \\
\hline ACEi/ARB & $81(37)$ & $80(35)$ & 0.55 \\
\hline Nitrate & $7(3)$ & $4(2)$ & 0.31 \\
\hline \multicolumn{4}{|l|}{ Presentation } \\
\hline Systolic blood pressure, $\mathrm{mmHg}$ & $128(110-144)$ & $128(110-148)$ & 0.73 \\
\hline Ejection fraction, $\%$ & $45(37-53)$ & $44(37-52)$ & 0.63 \\
\hline Creatinine, $\mathrm{mg} / \mathrm{dL}$ & $1(0.8-1.1)$ & $0.9(0.8-1.1)$ & 0.24 \\
\hline Haemoglobin, g/dL & $13.9(12.8-15)$ & $14.3(13-15.4)$ & 0.03 \\
\hline Time to reperfusion, $\mathrm{h}$ & $3.5(2.5-6.2)$ & $3.9(2.6-7)$ & 0.27 \\
\hline Anterior MI & $95(44)$ & $101(44)$ & 0.99 \\
\hline \multicolumn{4}{|l|}{ Killip class at admission } \\
\hline I & $184(85)$ & $203(88)$ & 0.36 \\
\hline II & $28(13)$ & $22(10)$ & \\
\hline III & $5(2)$ & $6(3)$ & \\
\hline
\end{tabular}

Data are presented as count (percentage) or median (interquartile range)

$A C E i / A R B$ angiotensin-converting enzyme inhibitor or angiotensin II receptor blocker, $B M I$ body mass index, $C A B G$ coronary artery bypass grafting, $I H D$ ischaemic heart disease, $M I$ myocardial infarction, $P C I$ percutaneous coronary intervention

Follow-up time for RIC and SOC was 2.1 (1.5-2.9) and 2.2 (1.5-3.0) years, respectively. Maximum follow-up time was 3.7 years in both groups. RIC showed lower hazard of combined cardiac mortality or hospitalization for $\mathrm{HF}$ $(\mathrm{HR}=0.35,95 \%$ CI $0.15-0.78, P=0.01)$ compared with SOC (Fig. 3a). In SOC 17 patients were readmitted due to HF on follow-up, their EF was 30 (25-34) and 7 eventually died from cardiac cause. Four other patients died from cardiac cause without prior admission. In RIC 8 patients were readmitted due to HF, their EF was 32 (29-36) and only 3 later died from cardiac cause. Six ICD devices were implanted in SOC and 3 in RIC. Planned ICD implantation without worsening $\mathrm{HF}$ clinic was documented in 4 and 3 patients in SOC and RIC. Excluding these cases from analysis did not change the observed differences for the combined outcome $(P<0.01)$. As for secondary outcome measures RIC showed lower hazards of cardiac mortality and hospitalization for HF but not for all-cause mortality and MACCE
(Fig. 3b). Causes of non-cardiac mortality were cancer (2 and 6 patients in RIC and SOC, respectively), stroke (5 patients in RIC), septic shock ( 2 and 1 patients in RIC and SOC, respectively), limb ischaemia or ischaemic colitis (2 patients in RIC) and suicide (1 patient in RIC). Five patients were found dead at home, 2 and 3 in RIC and SOC, respectively. Repeat MI on follow-up ensued in 7 and 5 patients in SOC and RIC, respectively. The NNT to prevent 1 event of combined outcome and 1 cardiac death at 2 years was 13 (9-40) and 26 (20-198), respectively, based on survival functions and HR [1].

As pre-specified in the study protocol, we carried out analyses for the main outcome in subgroups with (1) time from symptoms to reperfusion $>3 \mathrm{~h}$ (146 vs 131 patients in RIC and SOC, respectively; $P<0.01, \mathrm{HR}=0.2595 \% \mathrm{CI}$ $0.09-0.68),(2)$ anterior MI $(P=0.02, \mathrm{HR}=0.3595 \% \mathrm{CI}$ $0.15-0.85)$ and (3) totally occluded coronary artery (TIMI flow grade $0 ; P=0.04, \mathrm{HR}=0.4095 \% \mathrm{CI} 0.16-0.98$ ). 
Table 2 Procedural data from the catheterization laboratory

\begin{tabular}{|c|c|c|c|}
\hline & $\operatorname{SOC}(n=217)$ & $\operatorname{RIC}(n=231)$ & $P$ value \\
\hline \multicolumn{4}{|l|}{ Number of diseased vessels } \\
\hline 1 & $142(65)$ & $158(68)$ & 0.32 \\
\hline 2 & $40(18)$ & $55(24)$ & \\
\hline 3 & $34(16)$ & $18(8)$ & \\
\hline \multicolumn{4}{|l|}{ Culprit artery } \\
\hline Left anterior descending & $103(47)$ & $110(48)$ & 0.97 \\
\hline Circumflex & $27(12)$ & $36(16)$ & 0.34 \\
\hline Right coronary artery & $86(40)$ & $84(36)$ & 0.48 \\
\hline Angioplasty & $210(97)$ & $225(97)$ & 0.69 \\
\hline Stenting & $197(91)$ & $208(90)$ & 0.79 \\
\hline Thrombus aspiration & $71(33)$ & $91(39)$ & 0.14 \\
\hline \multicolumn{4}{|l|}{ TIMI flow grade } \\
\hline 0 & $150(69)$ & $170(74)$ & 0.38 \\
\hline 1 & $32(15)$ & $22(10)$ & \\
\hline 2 & $32(15)$ & $23(10)$ & \\
\hline 3 & $18(8)$ & $23(10)$ & \\
\hline \multicolumn{4}{|c|}{ TIMI flow grade after angioplasty } \\
\hline 0 & $3(1)$ & $5(2)$ & 0.91 \\
\hline 1 & $1(0)$ & $4(2)$ & \\
\hline 2 & $10(5)$ & $5(2)$ & \\
\hline 3 & $203(94)$ & $217(94)$ & \\
\hline
\end{tabular}

Data are presented as median (interquartile range)

TIMI, thrombolysis in myocardial infarction study group grading of coronary flow

\section{Discussion}

After promising results of RIC on myocardial salvage index after STEMI $[2,5]$ and post hoc analyses suggesting improved long-term clinical outcomes [21], a large size RCT addressing hard clinical endpoints has long been awaited [10]. So far RIC-STEMI is the largest RCT primarily evaluating the effect of RIC on long-term clinical outcomes after STEMI. RIC-STEMI shows that RIC as adjuvant to SOC reduces the combined event of cardiac mortality and hospitalization for HF after STEMI raising the hypothesis that it may be an effective mean to prevent progression to HF.

Although RIC was portended to reduce IRI and therefore progression towards HF after STEMI few studies actually addressed HF as outcome, and never as main outcome measure. Sloth et al. reported a trend for lower readmissions due to HF, 4 vs 7 cases in RIC and control for a median follow-up period of nearly 4 years [21] whereas Eitel et al. showed a trend for HF reduction at 6-months of follow-up: 4 cases of new onset HF in the RIC + post-conditioning group and 13 cases in the control group [5]. Two other smaller RCTs accounted for an additional 2 and 5 HF events in the RIC and control groups, respectively [16]. A recent meta-analysis which pooled data from the aforementioned studies reports an RR of 0.41 for HF reduction [18] which is interestingly very similar to our HR of 0.43 estimated for hospitalization due to HF. Subgroup analysis revealed even further differences in patients whose interval from symptom onset to reperfusion was longer than $3 \mathrm{~h}$. RIC-STEMI found differences in the main combined outcome of cardiac mortality and hospitalization for HF and in both as separate secondary outcomes, strongly supporting that RIC prevents progression to HF and cardiac-related death after STEMI. Further support to this hypothesis was derived from early in-hospital data and long-term follow-up EF outcomes. RIC patients were less likely to show signs of HF and to be treated with diuretics, inotropes or IABP during hospital stay and in the longer-run, although only a trend was found for the whole sample we newly report that RIC had a larger impact in EF recovery in STEMI patients presenting with poorer LV function. A $10 \%$ difference between groups was found for median EF 12 months after STEMI. This heterogeneous response may explain divergent outcomes from previous studies $[2,5,16]$ and partly corroborates reports of improved EF in RIC being restricted to patients with large anterior MI with left anterior descending artery as culprit artery $[2,19]$, although we did not find significant results in a similar subgroup analysis (data not shown). Most importantly follow-up was planned for 12 months and clearly documents long-term prevention of LV remodelling and dysfunction by RIC in the subgroup of STEMI patients with impaired LV function. Previous studies assessed EF at earlier time-points where the extent of recovery may have been hard to appraise due to variability in time to recovery between patients. Indeed, post-STEMI EF recovery only seems to stabilize beyond 6 months of follow-up [3]. Improved clinical outcomes and EF are at marked contrast with the absence of differences in troponin I levels between RIC and SOC. Indeed, Botker et al. already failed to show reduced infarct size through troponin levels in RIC patients in their seminal study despite the demonstration of enhanced myocardial salvage index at 30 days [2]. This could be due to the high variability in biomarker levels as a consequence of patient heterogeneity regarding coronary territories involved and duration of ongoing ischaemia. Moreover, it is also likely that systemic influences from RIC improve myocardial function and remodelling by targeting signalling pathways in the myocardium at risk or in the remote myocardium $[12,14]$. 

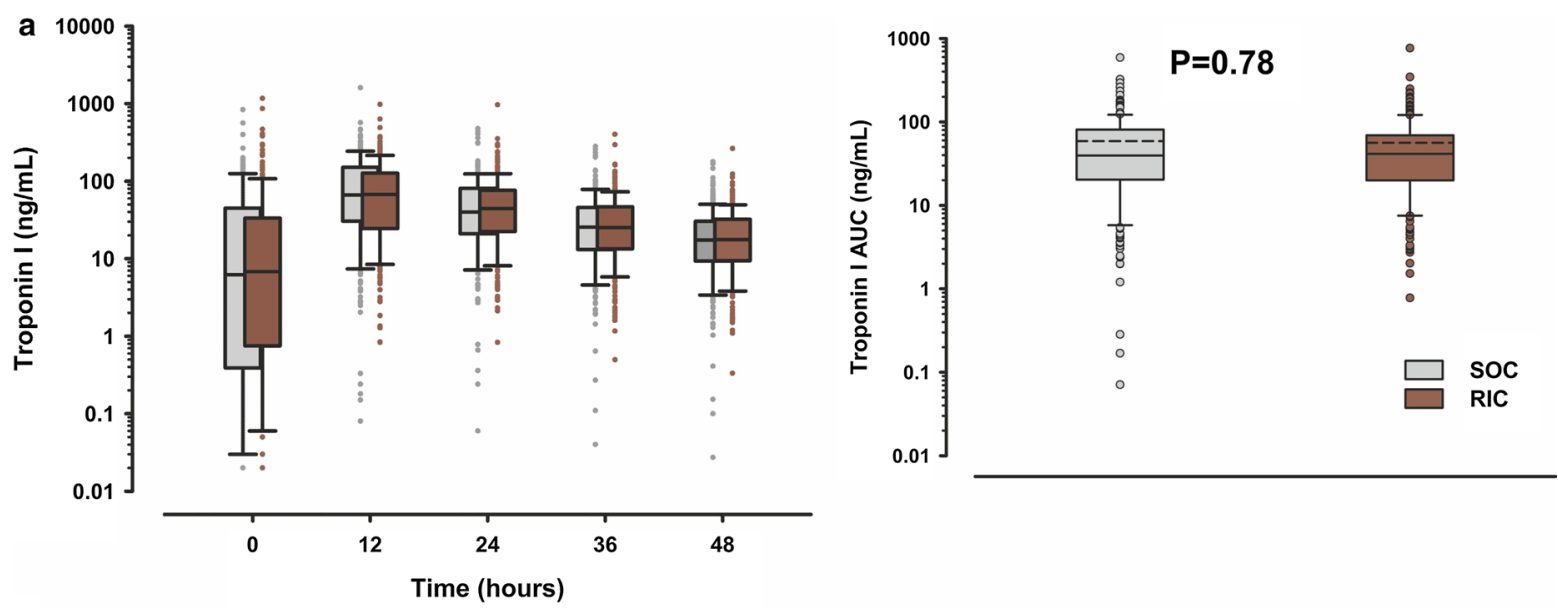

b
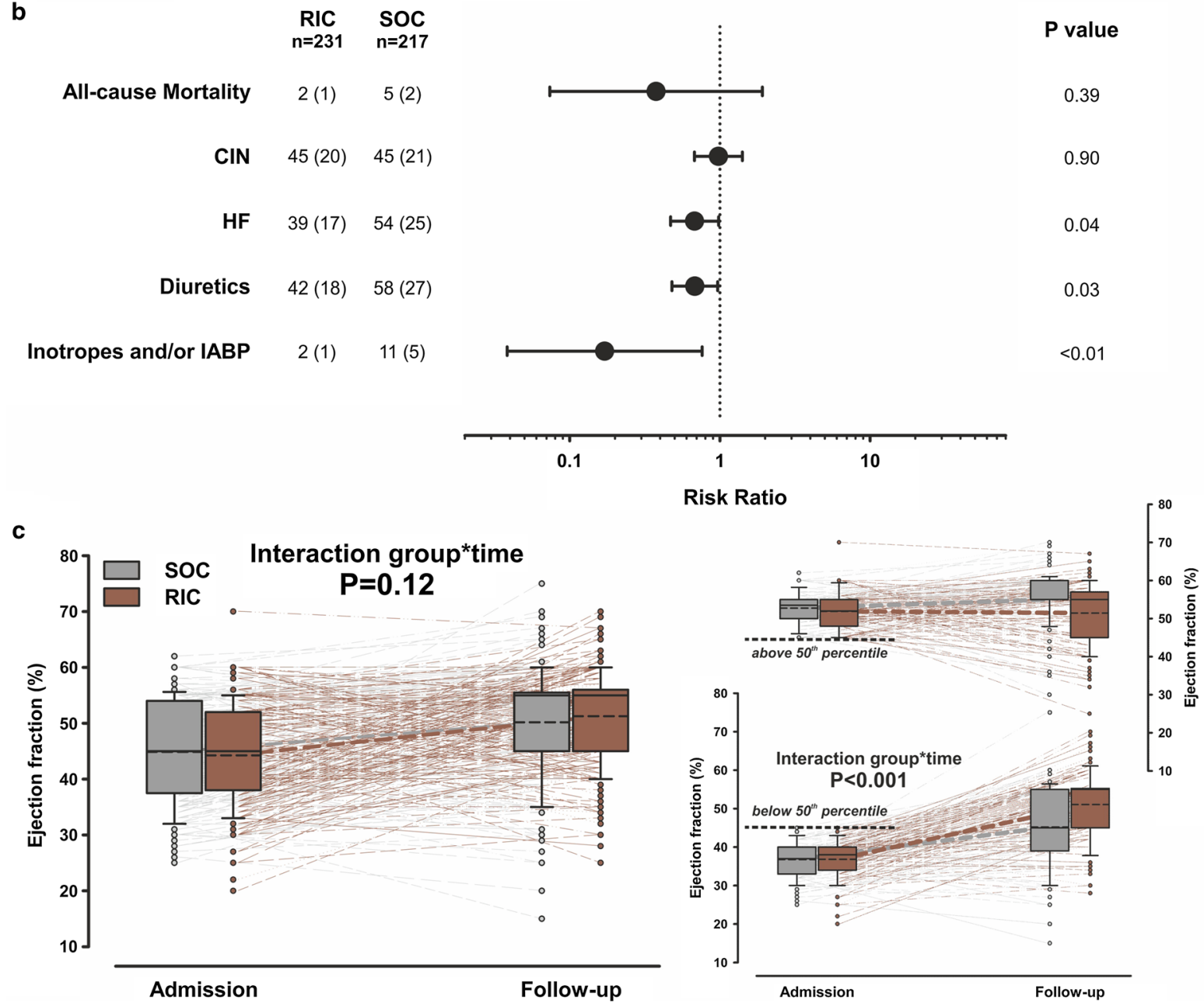

Fig. 2 Evolution of troponin I serum levels and corresponding area under curve (AUC) (a), main outcomes during hospital stay (b) and evolution of ejection fraction as of 12 months after ST-elevation myocardial infarction (c) in patients allocated to remote ischaemic conditioning (RIC) or standard of care (SOC). In b risk ratios and corresponding $95 \%$ confidence intervals are plotted alongside number (percentage) of events and corresponding $P$ values. SOC was taken as reference. In c paired box-plots and detailed before-after line plots are given for ejection fraction at admission and follow-up $(n=173$ and 193 for SOC and RIC, respectively), the mean is represented by dashed lines. Alongside, groups are equally divided in 2-quantiles (median is $45 \%$, dashed lines) according to admission EF denoting a strong interaction in the lower EF group 
Fig. 3 Cumulative hazard plots for the main outcome measure of combined cardiac death and hospitalization for heart failure (a) and hazard ratios plots for secondary outcome measures (b) in patients allocated to remote ischaemic conditioning (RIC) or standard of care (SOC). In a life-table analysis with detailed number of presented beneath the cumulative hazard and corresponding 95\% confidence interval plot and respective $P$ value for group comparisons. In $\mathbf{b}$ hazard ratios and corresponding 95\% confidence intervals are plotted alongside number (percentage) of events and corresponding $P$ values. SOC was taken as reference patients at risk in each group is

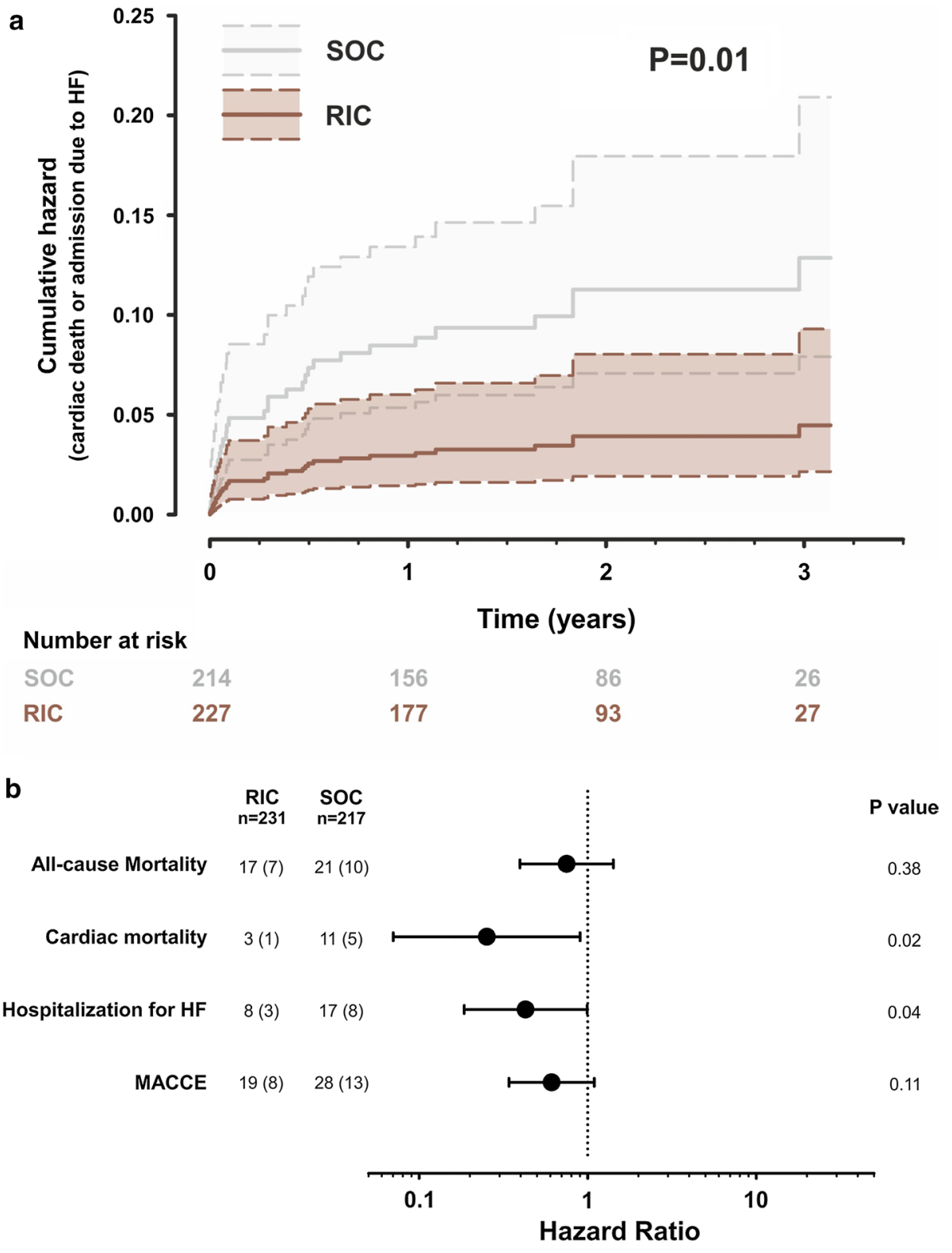

In contrast to the CONDI sub-trial [21] which found reduction in MACCE or all-cause mortality in RIC as outcomes (though originally powered for myocardial salvage index), but in keeping with the larger LIPSIA CONDITIONING trial [5] we found only a trend towards reduced MACCE on follow-up after RIC and no difference in allcause mortality. This could be partly ascribed to the longer follow-up period of CONDI as well as to random factors. As reported in previous studies a wide within-group dispersion and no differences between groups were found for AUC and peak troponin I serum levels, partly owing to variability in time from symptom onset to PCI and diversity of clinical profiles of STEMI patients enrolled [2, 5]. An unexpected finding was increased haemoglobin concentrations in RIC. This previously unappreciated phenomenon is unlikely to be a chance finding and might be explained by the fact that blood sample collection took place during or soon after tourniquet application. As reported in healthy athletes, tourniquet progressively raises haemoglobin levels [15]. Whether raised haematocrits play a role in conditioning remains to be defined. Interestingly, low-dose haemoglobin-based oxygen carriers were protective in a dog model of cardiac IRI [25].

One of the major issues that can be raised regarding study design is deviation from an intention-to-treat approach (ITT). Nevertheless, we must argue that intervention was a 30-min protocol entirely on the hands of health care providers, added little or no risk or further complexity to cardiac catheterization, was well tolerated and has no known untoward side effects. For these reasons no patient crossedover to the other study group, all patients complied with the 
intervention and none withdrew from the study. The logic behind ITT is precisely to account for these events when compliance is poor [20]. Moreover, allocation and intervention were done in an emergency setting before a definite diagnosis or all exclusion criteria could be ascertained. A high proportion of patients from each group (10 and 15\%) interrupted the intervention shortly after allocation and before completion of the protocol, as soon as misdiagnosis or exclusion criteria were recognized. In our view, knowing what would have happened to these patients on follow-up is beside the point because in clinical practice the intervention would have also been interrupted anyway. Treatment was not intended for these subjects. We faced these cases as flunked allocation. The purposes of randomization were not violated and bias should be minimal. Likewise, 2 additional patients were later found to be emigrants briefly staying in the country for holidays. No further data could be retrieved beyond discharge, all major outcomes were unavailable and imputation was not considered an option. In a broader framework of modified ITT we must underscore that all outcome measures were available for the remaining patients except for follow-up echocardiography data, due to earlier mortality or missing data (13\%). Indeed, we found a trend towards higher drop-out in SOC compared with RIC ( 29 vs $22 \%$, respectively, $P=0.09$ ) which may have partly biased results. Still, we were forced to work with existing data since paired analysis was envisaged. Another important concern is that sample size estimates for the main outcome were partly flawed. The lower rates of combined outcome and higher than expected rate of unconfirmed STEMI reduced power which was counterbalanced by the lower than predicted number of drop-outs and losses to follow-up and the higher than predicted follow-up time per individual. Estimated power was $75 \%$. Despite the limitations of post hoc power analysis we must underscore that significant differences were found between groups, results were robustly significant and 95\% CI were not wide, suggesting that the study was adequately powered [23]. Finally, blinding is currently not feasible in RIC RCTs because operation of cuff devices cannot be concealed from either patients or attending physicians. Procedures in the catheterization laboratory were not controlled for. Although no significant differences were found between groups, there was a trend for higher rate of thrombus aspiration in RIC. Lastly, due to the organization of the Portuguese health system admissions due to HF were not restricted to our centre and therefore the implementation of an independent committee to oversee HF admissions was not viable. Measures were undertaken to avoid further bias during outcome assessment and statistical analysis.

Despite recent disappointing outcomes with the postconditioning strategy [5] and in the cardiac surgery setting [9] which may be explained by the negative influence of propofol in RIC since other authors have reported improved long-term survival after coronary artery bypass grafting [13], we have shown in the largest RCT to date powered to assess a hard combined clinical endpoint as main outcome that RIC as adjunctive to SOC reduces cardiac mortality and hospitalization for HF after STEMI. We have also shown enhanced recovery of EF on 12 months follow-up in patients presenting with STEMI and impaired LV function. Still we must underscore that this is a single-centre study with limited sample size, results should be confirmed in larger multicentre studies.

Acknowledgements We would like to thank all the nurses and interventional cardiologists engaged in the study at Hospital de Braga.

Funding This work was supported by the Portuguese Foundation for Science and Technology (Projects PEst-C/SAU/UI0051/2011 and EXCL/BIM-MEC/0055/2012) through the Cardiovascular R\&D Unit, by the European Commission Grant FP7-Health-2010 (MEDIA261,409) and by Project DOCnet (NORTE-01-0145-FEDER-000003), supported by Norte Portugal Regional Operational Programme (NORTE 2020), under the PORTUGAL 2020 Partnership Agreement, through the European Regional Development Fund (ERDF). European Structural and Investment Funds (ESIF), under Lisbon Portugal Regional Operational Programme and National Funds through Foundation for Science and Technology under project POCI-01-0145FEDER-016385. This work was supported by a grant from José de Mello Saúde.

\section{Compliance with ethical standards}

Conflict of interest The authors declare that they have no conflict of interest.

\section{References}

1. Altman DG, Andersen PK (1999) Calculating the number needed to treat for trials where the outcome is time to an event. Br Med J 319:1492-1495

2. Botker HE, Kharbanda R, Schmidt MR, Bottcher M, Kaltoft AK, Terkelsen CJ, Munk K, Andersen NH, Hansen TM, Trautner S, Lassen JF, Christiansen EH, Krusell LR, Kristensen SD, Thuesen L, Nielsen SS, Rehling M, Sorensen HT, Redington AN, Nielsen TT (2010) Remote ischaemic conditioning before hospital admission, as a complement to angioplasty, and effect on myocardial salvage in patients with acute myocardial infarction: a randomised trial. Lancet 375:727-734. https://doi.org/10.1016/S0140-6736(09)62001-8

3. Cao F, Sun D, Li C, Narsinh K, Zhao L, Li X, Feng X, Zhang J, Duan Y, Wang J, Liu D, Wang H (2009) Long-term myocardial functional improvement after autologous bone marrow mononuclear cells transplantation in patients with ST-segment elevation myocardial infarction: 4 years follow-up. Eur Heart J 30:1986-1994. https ://doi.org/10.1093/eurheartj/ehp220

4. Eagle KA, Nallamothu BK, Mehta RH, Granger CB, Steg PG, Van de Werf F, Lopez-Sendon J, Goodman SG, Quill A, Fox KA (2008) Trends in acute reperfusion therapy for ST-segment elevation myocardial infarction from 1999 to 2006: we are getting better but we have got a long way to go. Eur Heart J 29:609-617. https://doi. org/10.1093/eurheartj/ehn069

5. Eitel I, Stiermaier T, Rommel KP, Fuernau G, Sandri M, Mangner N, Linke A, Erbs S, Lurz P, Boudriot E, Mende M, Desch S, Schuler G, 
Thiele H (2015) Cardioprotection by combined intrahospital remote ischaemic perconditioning and postconditioning in ST-elevation myocardial infarction: the randomized LIPSIA CONDITIONING trial. Eur Heart J 36:3049-3057. https://doi.org/10.1093/eurheartj/ ehv463

6. Fox KA, Carruthers KF, Dunbar DR, Graham C, Manning JR, De Raedt H, Buysschaert I, Lambrechts D, Van de Werf F (2010) Underestimated and under-recognized: the late consequences of acute coronary syndrome (GRACE UK-Belgian Study). Eur Heart J 31:2755-2764. https://doi.org/10.1093/eurheartj/ehq326

7. Frohlich GM, Meier P, White SK, Yellon DM, Hausenloy DJ (2013) Myocardial reperfusion injury: looking beyond primary PCI. Eur Heart J 34:1714-1722. https://doi.org/10.1093/eurheartj/eht090

8. Gaspar A, Pereira MA, Azevedo P, Lourenco A, Marques J, LeiteMoreira A (2015) Remote ischemic conditioning in ST-elevation myocardial infarction as adjuvant to primary angioplasty (RICSTEMI): study protocol for a randomized controlled trial. Trials 16:398. https://doi.org/10.1186/s13063-015-0937-1

9. Heusch G (2016) The coronary circulation as a target of cardioprotection. Circ Res 118:1643-1658. https://doi.org/10.1161/CIRCR ESAHA.116.308640

10. Heusch G (2017) Critical issues for the translation of cardioprotection. Circ Res 120:1477-1486. https://doi.org/10.1161/CIRCR ESAHA.117.310820

11. Heusch G, Gersh BJ (2017) The pathophysiology of acute myocardial infarction and strategies of protection beyond reperfusion: a continual challenge. Eur Heart J 38:774-784. https://doi. org/10.1093/eurheartj/ehw224

12. Heusch G, Rassaf T (2016) Time to give up on cardioprotection? A critical appraisal of clinical studies on ischemic pre-, post-, and remote conditioning. Circ Res 119:676-695. https://doi.org/10.1161/ CIRCRESAHA.116.308736

13. Kleinbongard P, Peters J, Jakob H, Heusch G, Thielmann M (2018) Persistent survival benefit from remote ischemic pre-conditioning in patients undergoing coronary artery bypass surgery. J Am Coll Cardiol 71:252-254. https://doi.org/10.1016/j.jacc.2017.10.083

14. Kleinbongard P, Skyschally A, Heusch G (2017) Cardioprotection by remote ischemic conditioning and its signal transduction. Pfluegers Arch: Eur J Physiology 469:159-181. https://doi.org/10.1007/ s00424-016-1922-6

15. Kuipers H, Brouwer T, Dubravcic-Simunjak S, Moran J, Mitchel D, Shobe J, Sakai H, Stray-Gundersen J, Vanhoutvin S (2005) Hemoglobin and hematocrit values after saline infusion and tourniquet. Int J Sports Med 26:405-408. https://doi.org/10.1055/s-2004-821156

16. Liu Z, Zhao L, Hong D, Gao J (2016) Remote ischaemic preconditioning reduces myocardial ischaemic reperfusion injury in patients with ST-elevation myocardial infarction undergoing primary percutaneous coronary intervention. Acta Cardiol 71:596-603. https ://doi.org/10.2143/ac.71.5.3167504

17. Mamas MA, Anderson SG, O'Kane PD, Keavney B, Nolan J, Oldroyd KG, Perera D, Redwood S, Zaman A, Ludman PF, de Belder MA (2014) Impact of left ventricular function in relation to procedural outcomes following percutaneous coronary intervention: insights from the British Cardiovascular Intervention Society. Eur Heart J 35:3004-3012a. https://doi.org/10.1093/eurheartj/ehu303

18. McLeod SL, Iansavichene A, Cheskes S (2017) Remote ischemic perconditioning to reduce reperfusion injury during acute STsegment-elevation myocardial infarction: a systematic review and meta-analysis. J Am Heart Assoc. https://doi.org/10.1161/ JAHA.117.005522

19. Munk K, Andersen NH, Schmidt MR, Nielsen SS, Terkelsen CJ, Sloth E, Botker HE, Nielsen TT, Poulsen SH (2010) Remote ischemic conditioning in patients with myocardial infarction treated with primary angioplasty: impact on left ventricular function assessed by comprehensive echocardiography and gated singlephoton emission CT. Circ Cardiovasc Imag 3:656-662. https://doi. org/10.1161/circimaging.110.957340

20. Shrier I, Steele RJ, Verhagen E, Herbert R, Riddell CA, Kaufman JS (2014) Beyond intention to treat: what is the right question. Clin Trials (Lond, Engl) 11:28-37. https://doi.org/10.1177/1740774513 504151

21. Sloth AD, Schmidt MR, Munk K, Kharbanda RK, Redington AN, Schmidt M, Pedersen L, Sorensen HT, Botker HE, Investigators C (2014) Improved long-term clinical outcomes in patients with ST-elevation myocardial infarction undergoing remote ischaemic conditioning as an adjunct to primary percutaneous coronary intervention. Eur Heart J 35:168-175. https://doi.org/10.1093/eurheartj/ eht369

22. Townsend N, Nichols M, Scarborough P, Rayner M (2015) Cardiovascular disease in Europe 2015: epidemiological update. Eur Heart J 36:2673-2674

23. Lenth RV (2007) Post Hoc power: tables and commentary

24. Wobbrock JO, Findlater L, Gergle D, Higgins JJ (2011) The aligned rank transform for nonparametric factorial analyses using only anova procedures. In: Proceedings of the SIGCHI conference on human factors in computing systems. ACM, Vancouver, BC, Canada, $p$ 143-146

25. Yang Q, Wu W, Li Q, Chen C, Zhou R, Qiu Y, Luo M, Tan Z, Li S, Chen G, Zhou W, Liu J, Yang C, Liu J, Li T (2015) Highdose polymerized hemoglobin fails to alleviate cardiac ischemia/ reperfusion injury due to induction of oxidative damage in coronary artery. Oxid Med Cell Longevity 2015:125106. https://doi. org/10.1155/2015/125106 